TERRA. Revista de Desarrollo Local e-ISSN: 2386-9968

Número 6 (2020), 271-293

DOI 10.7203/terra.6.16708

IIDL - Instituto Interuniversitario de Desarrollo Local

\title{
Las mujeres como agentes para la cohesión territorial de los destinos turísticos rurales
}

\author{
César García Pina \\ Doctorando Escuela Internacional de Doctorado (Universidad de Murcia, España) \\ cesar.garcia1@um.es \\ https://orcid.org/0000-0002-0638-3415
}

\section{María del Carmen Solano Báez}

Observatorio de Desarrollo Rural Local y Empleo (Murcia, España)

carmen.solano@observalocal.com

https://orcid.org/0000-0003-4431-9956

\section{Prudencio José Riquelme Perea}

Prof. Titular de la Facultad de Ciencias del Trabajo (Universidad de Murcia, España) halconps@um.es

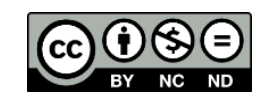

Esta obra se distribuye con la licencia Creative Commons Reconocimiento-NoComercial-SinObraDerivada 4.0 Internacional 


\title{
SECCIÓN NOTAS Y AVANCES DE INVESTIGACIÓN
}

\author{
Las mujeres como agentes para la cohesión territorial de los destinos turísticos rurales
}

Resumen. Las mujeres rurales, presentes, pero invisibles en los procesos emergentes del medio rural, se revelan como colectivo impulsor para la reconstrucción de los territorios rurales. Este segmento de la población promueve procesos creativos que dinamizan el mundo rural e instrumenta estrategias de diversificación necesarias para sostener la vida y el territorio. Estas estrategias dan lugar a la diversificación de las funciones productivas del territorio y posibilitan la creación de destinos turísticos rurales sostenibles y de enfoque territorial. No obstante, no se muestran modificaciones significativas en la división del trabajo por género. A través de un análisis cualitativo, realizado bajo los principios metodológicos de la Teoría Fundamentada, se elabora un marco explicativo acerca del rol de la mujer en la esfera pública de los destinos turísticos rurales. Así, tras analizar la realidad murciana en particular -y la española, mexicana y tailandesa en general-, los patrones de respuesta muestran una prevalencia de los roles de género para sostener el territorio y favorecer su transición a destino. En la configuración del destino turístico, la mujer es percibida como facilitadora para el diseño de actuaciones transversales.

Palabras clave. Mujer rural, ecofeminismo, desarrollo rural territorial, destinos turísticos rurales.

\section{Women as agents for territorial cohesion in rural tourism destinations}

\begin{abstract}
Rural women, present but invisible in the emerging processes of the rural environment, are revealed as a driving group for the reconstruction of rural territories. This sector of the population promotes creative processes that energize the rural world and implement diversification strategies necessary to sustain life and the territory. These strategies lead to the diversification of the territory's productive functions and the creation of sustainable rural tourism destinations with a territorial approach. However, they do not show significant changes in the gender division of labour. Through a qualitative analysis carried out under the methodological principles of the Grounded Theory, an explanatory framework is constructed about the role of women in the public sphere of rural tourist destinations. Thus, after analysing the reality of Murcia in particular and the Spanish, Mexican and Thai reality in general, the response patterns show a prevalence of gender roles to sustain the territory and enable its transition to the destination. In the configuration of the tourist destination, women are perceived as facilitators for the design of transversal actions.
\end{abstract}

Keywords: Rural women, ecofeminism, territorial rural development, rural tourist destinations.

Recibido: 19 de febrero de 2020

Devuelto para revisión: 27 de marzo de 2020

Aceptado: 27 de abril de 2020

\section{Referencia / Citation:}

García, C., Solano, M. C., y Riquelme, P. J. (2020). Las mujeres como agentes para la cohesión territorial de los destinos turísticos rurales. TERRA. Revista de Desarrollo Local, (6), 271-293. DOI 10.7203/terra.6.16708 


\section{INTRODUCCIÓN}

La agricultura europea ha afrontado diferentes cambios socioeconómicos que modifican la estructura productiva del territorio. Esto es especialmente sensible en el medio rural, que ha pasado de una economía fundamentalmente agrícola a una más terciarizada (Dessein et al., 2013; Camarero, 2017a). Estas transformaciones han generado desigualdades en la estructura social de los territorios. Así, a medida que se ha ido incrementando la modernización del sector agrícola, se ha profundizado en la masculinización del territorio y, por tanto, la exclusión de la mujer (Seuneke y Bock, 2015).

Ante esa estructura patriarcal en la agricultura y ganadería familiar (Brandth, 2002), la mujer rural ha protagonizado importantes procesos de innovación social. Con ello, ha favorecido la existencia de emprendimientos multifuncionales donde aparecen nuevas actividades, productos y servicios (Seuneke y Bock, 2015). De esta manera, se han desarrollado actividades como el turismo rural y se han propiciado nuevos espacios de aprendizaje y cooperación, pero sin una modificación sustancial en los roles de género (Annes y Wright, 2015).

En este contexto de innovación social, surgen iniciativas dirigidas a incrementar el protagonismo de la mujer en la agenda política (Shortall, 2015) mejorando su posición en lo referente a la gestión y titularidad de la tierra. También se busca, por parte de los responsables políticos, minimizar algunos de los efectos negativos derivados de una estructura agraria patriarcal, caracterizada por la construcción de la identidad masculina a partir de una fuerte relación de poder sobre los medios de producción y el propio territorio (Little y Panelli, 2003; Brandth y Haugen, 2010; Little, 2015). En la actualidad se mantiene una importante barrera laboral de género en materia agrícola (Shortall et al., 2020). No obstante, otros autores defienden que esta barrera se ha suavizado con la promoción de la multifuncionalidad en la agricultura (Seuneke y Bock, 2015).

De acuerdo con Woods (2005), las zonas rurales siempre han sido espacios de ajuste, afectados por la modernización tecnológica y social, así como por la globalización económica. Todo ello ha modificado sustancialmente la fisonomía del territorio. Durante la década de años los noventa el auge de la multifuncionalidad de la agricultura abrió la posibilidad a las mujeres rurales de integrar nuevas actividades en la unidad de producción familiar. También permitió desarrollar una nueva identidad profesional a partir del surgimiento de los empresarios rurales (Dessein et al., 2013). En el caso español, es la década que marcó la llegada del turismo rural, financiado por LEADER. Este instrumento de financiación se ha constituido como la principal vía para la diversificación económica a través de la actividad turística (Sarasa, 2000; García Marín, 2011; Pulido y Cárdenas, 2011).

El desarrollo de la multifuncionalidad agrícola de los años 90 (Seuneke y Bock, 2015) y la llegada del turismo como una actividad de diversificación para la economía rural en general (Pulido y Cárdenas, 2011), y para la mujer en particular (Alonso y Trillo, 2014), ha facilitado la definición de nuevas identidades (Brandth, 2010). Pero las barreras de acceso a espacios de decisión de la agricultura, la persistencia de los roles de género en la actividad turística y la masculinización demográfica han afectado en la definición de los territorios y la configuración de los destinos turísticos rurales.

En este sentido, la decisión final sobre la puesta en funcionamiento de la actividad turística depende, en última instancia, de la aprobación masculina. También, debe 
mantener los roles de género que refuerzan el buen funcionamiento agrícola por lo que no promueve el empoderamiento de la mujer rural (Annes y Wright, 2015). Desde el punto de vista de Brandth y Haugen (2012), el desarrollo de iniciativas agroturísticas podría favorecer el reposicionamiento de la mujer, pasando de un espacio invisible a un rol protagónico en la estructura familiar. Pero siempre, gracias a la labor de armonización de la mujer rural, entre las actividades remuneradas y el trabajo para sostener las dinámicas de la vida familiar (Rijkers y Costa, 2012).

En la evolución del discurso de la literatura en el ámbito del turismo rural y de variantes como el agroturismo, ganan importancia los enfoques integrales de planificación ascendente (Camarero y Oliva, 2013). La literatura más actual, sugiere que el turismo en el medio rural contribuye a la cohesión social y al desarrollo económico del territorio (Brandth y Haugen, 2012; Pulido y Parrilla, 2016). Esto permite identificar las medidas emprendidas por las mujeres para articular la familia, la unidad de producción y el territorio (Little y Panelli, 2003). Es decir, para sostener la vida el territorio y posibilitar su transición a destino turístico. Ya que, es a nivel destino, donde los agentes del territorio y los turistas dan nuevos significado a los lugares.

Por ello, el estudio del rol de la mujer en un destino de configuración territorial muestra otras dimensiones que resaltan el papel desempeñado por la mujer. Moisy (2001), Saarinen (2004), Saraniemi y Kylänen (2011), y Blasco et al. (2013), coinciden en la idoneidad del destino como unidad de análisis para un estudio integral de la actividad turística. Desde este plano es posible ver en qué aspectos se mantienen los roles de género y qué condiciones específicas son necesarias para el sostenimiento de las tres dimensiones en las que está inmersa la mujer: la familia, el territorio y el destino. A partir de lo anterior, la línea de investigación de este artículo se centra en el protagonismo de la mujer como piedra angular para el desarrollo territorial y la mejora de los procesos del destino turístico. Las conclusiones confluyen con las visiones arrojadas por dos perspectivas teóricas: el desarrollo territorial y el ecofeminismo.

Por un lado, el desarrollo territorial hace énfasis en el territorio como una construcción (Pequeur, 2005) que moviliza a los agentes para la construcción de una estrategia territorial (Ramos y Garrido, 2011). Así, el territorio se convierte en un lugar de desarrollo multidimensional (Alburquerque, 2001; Vázquez-Barquero, 2007, 2018) con una visión longitudinal. A partir de estas nuevas configuraciones, los territorios se redefinen o refundan "para dar lugar a nuevas identidades espaciales portadoras de nuevos medios de desarrollo cultural, social y económico" (Berdulay, 2009, p.19).

Por otro lado, el ecofeminismo, se centra en la amplitud de los procesos y trabajos necesarios para sostener la vida (Pérez, 2014) en el medio rural. Desde la perspectiva de la mujer rural, muestra su labor en los procesos productivos y sociales del territorio (Shiva, 1998). Al mismo tiempo, trae al debate la noción de la naturaleza (Herrero, 2015), un recurso esencial para el turismo y que requiere de otros enfoques para su análisis. Esta perspectiva permite analizar el sistema de valores y estructuras necesarias para el funcionamiento de la economía rural (Pérez, 2014; Shiva y Mies, 2016).

El artículo se organiza en cuatro apartados. Primero se presenta la literatura que aborda tres conceptos clave desde un enfoque territorial: mujer rural, territorio y destino turístico rural. En el segundo apartado, se explica la metodología utilizada, partiendo de los principios de la Teoría Fundamentada de tipo glaseriano, seguido del método para el triple proceso de codificación y la estructuración del esquema teórico que ordena el informe final. En el tercer apartado, se presenta el informe de resultados de la investigación en un diálogo con la literatura citada, acorde con los criterios de la Teoría Fundamentada que 
introduce la literatura como una estrategia de confirmación para el informe final. Por último, el artículo finaliza con el apartado de conclusiones que incorpora los hallazgos más relevantes de la investigación.

\section{LA MUJER RURAL, EL TERRITORIO Y LOS DESTINOS TURÍSTICOS}

La línea de investigación de este artículo y que, consecuentemente, articula el marco teórico, analiza las tres dimensiones en las que está inmersa la mujer rural: la familia, el territorio y el destino. Esta triada, definida a partir del enfoque territorial, lleva implícitos tres elementos que ayudan a comprender mejor los procesos que tienen lugar: proporciona centralidad a los actores, una visión longitudinal del territorio y ofrece una análisis ascendente del destino turístico. Asimismo, debido a la naturaleza inductiva de la metodología empleada, la estructura del marco teórico se concentra en situar el objeto de estudio para responder a los objetivos de la investigación (Carrero et al., 2012), siendo este aspecto crucial ya que condiciona la estructura general del artículo.

En términos de "lo rural" el territorio adquiere una visión multidimensional, como un elemento para la construcción de identidad (Little y Panelli, 2003; Cánoves et al., 2014), de la actividad económica (Santos y Lois, 2015) y la articulación de las funciones sociales (Rubio y Mazón, 2009) y productivas del territorio (Vázquez-Barquero, 2017). Con base en estas funciones sociales, los actores establecen relaciones que contribuyen a generar una sensación de pertenencia y una alta implicación a partir de relaciones de respeto a la diversidad cultural y étnica (Carpio, 2000). Sin embargo, el territorio rural se asocia con un contexto con limitadas posibilidades para el desarrollo de la actividad económica, en declive, aislado, envejecido, y poco atractivo para desarrollar opciones vitales (Baylina, 2019; Camarero, 2009).

La multidimensión del territorio, desde la óptica del desarrollo territorial, añade complejidad a su estudio, ya que, desde esta visión, "el territorio significa una construcción social para el desarrollo territorial” (Ramos y Garrido, 2011, p.37). Además, "representa una agrupación de relaciones sociales, el lugar donde la cultura local y otros rasgos se han sedimentado" (Garofoli, 1991, p.56). Autores como Linck (2014) asocian la construcción del territorio a parte de la construcción de una estrategia territorial, lo cual permite compartir rasgos comunes que forman parte de la memoria colectiva de los agentes del territorio.

Pecqueur (2005), ahondando en la idea de territorio construido, los diferencia de los territorios dados, e identifica a los territorios como construcciones sociales a partir de la cultura y los valores comunes que identifican por sí mismos los actores y que solo ocurren gracias a su organización e interrelación. De esta forma, el territorio adquiere una perspectiva longitudinal debido a que se encuentra en continua evolución gracias a las dinámicas de los actores y la estrategia territorial adoptada para hacer frente a los efectos de ámbito global. Así, el paradigma del desarrollo territorial posibilita que se conceptualice el territorio como un patrimonio localizado (Link, 2014) que puede ser la base para el diseño de estrategias territoriales endógenas que reduzcan los desequilibrios de la globalización (Vázquez-Barquero, 2017, 2018). Por esta razón, el territorio se comprende como una vía para la comprensión integral de la realidad y se exhorta al rechazo de las nociones que banalizan tanto la definición como la gestión del territorio rural (Guinjoan et al., 2016). 
Por otro lado, cuando el territorio se contempla como un destino turístico, se concibe como un espacio que ha pasado por un proceso de homogeneización. Desde la perspectiva sociológica de Saarinen (2014) los destinos son dinámicos y adquieren personalidad gracias a su historia y cultura. De manera que, esta configuración, al margen del territorio, difumina sus especificidades territoriales y modifica el carácter autóctono de las actividades tradicionales, orientándose hacia una gestión centrada en la satisfacción de los turistas. Esta construcción del destino, a nivel subjetivo, está marcada por las expectativas de los visitantes y ello conduce a una vulgarización del destino (Brandth y Haugen, 2012, 2014; Camarero y Oliva, 2013). En la actualidad, aun cuando no existe un consenso sobre la definición de un destino, Dredge (1999), Moisy (2001), Saarinen (2004), Saraniemi y Kylänen (2011) y Blasco et al. (2013), coinciden en la pertinencia del destino como elemento de análisis para el estudio integral de la actividad turística.

A pesar de la fragilidad del territorio rural, se han desarrollado iniciativas dirigidas a su dinamización económica a partir del turismo (Pulido y Cárdenas, 2011). Estas iniciativas suelen utilizar el medio rural como soporte para el producto turístico. Por lo que su importancia se reduce a un valor basado en sus posibilidades de uso para la generación de oferta turística. Recientemente, después de 30 años de enfoque territorial en el turismo financiado por LEADER, se empiezan a dar cambios para comprender el destino desde el territorio, alejándose de la idea del espacio. Incluso se ha planteado analizar los destinos a partir de un enfoque cultural e integral, analizando la relación sujeto-objeto, es decir, de los turistas con los recursos turísticos del destino (Saraniemi y Kylänen, 2011). Así, desde esta perspectiva, se entiende que el destino evoluciona como resultado de la combinación de las dimensiones sociales, culturales, políticas y económicas (Saarinen, 2004).

Con una visión dinámica, tanto del destino como del territorio, mediante el enfoque territorial se sitúa en el centro a los agentes del territorio y, concretamente en esta investigación, a la mujer rural. La mujer rural lidera los procesos de cambio que permiten una transición hacia actividades no agrícolas como el turismo rural (Brandth y Haugen, 2007; Anns y Wright, 2015). De acuerdo con el Observatorio de Turismo Rural, España registra en 2019 un $58 \%$ de mujeres como propietarias de establecimientos rurales, esto evidencia cómo dicho colectivo ha progresado en este campo. Por esta razón, la Política de Desarrollo Rural del periodo de programación 2014-2020 señala a la mujer como agente prioritario de financiación, promueve la titularidad compartida para la inserción de la mujer en la agricultura y a través de LEADER, instrumentaliza el turismo rural.

La mujer rural es responsable de generar procesos de innovación mediante la incorporación de nuevas prácticas. Como protagonista de la multifuncionalidad (Seuneke y Bock, 2015) es un pilar para la reconstrucción de territorios que oscilan entre procesos de declive y procesos de construcción de estrategias para la dinamización territorial (Baylina, 2019). La transversalidad de las acciones de la mujer rural en el seno del territorio conlleva que su análisis se realice desde una perspectiva holística (Little y Panelli, 2003). para situar y definir el rol de la mujer en el proceso de configuración o reconfiguración de destinos turísticos rurales.

Este colectivo ha contribuido invisiblemente a la construcción de destinos turísticos, siendo un agente imprescindible de las políticas rurales, aunque vulnerable por el tipo de medio en el que desarrolla su trabajo (Alonso y Trillo, 2014). El aumento de las actividades del sector terciario, a la par de la mejora de la cualificación de las mujeres rurales, facilitó su incorporación al trabajo remunerado. Estos cambios fueron especialmente visibles en la década de los noventa (Brandth, 2002). Así, mientras que la agricultura mostraba una pronunciada tendencia hacia la masculinización, en ese mismo periodo la mujer inició un proceso de armonización entre las actividades remuneradas y 
el trabajo para sostener las dinámicas de la vida familiar (Annes y Wright, 2015; Rijkers, y Costa, 2012).

Si bien las investigaciones reconocen la labor de la mujer para el desarrollo de la multifuncionalidad agrícola (Seuneke y Bock, 2015), esta queda limitada por los roles de género y las estructuras sociales del medio rural (Brandth, 2002; Little, 2002). En la actualidad, el medio rural continúa siendo hostil para las mujeres (Little, 2015), provocando la persistencia del éxodo rural femenino (Baylina, 2019) y que algunas de las tareas más exigentes, como el cuidado a personas dependientes, se mantenga a cargo de las mujeres (Camarero y Sampedro, 2016). De igual manera, se mantiene la segregación y segmentación por género en el mercado laboral rural (Shortall et al., 2020). Es decir, la concentración de mujeres en trabajos catalogados como de mujeres, caracterizados por salarios bajos y limitado desarrollo de la carrera profesional elegida.

Desde una mirada positiva, la exclusión de la mujer en la agricultura ha favorecido su habilidad para desarrollar una identidad empresarial. En este sentido se han realizado estudios para identificar el proceso de aprendizaje en emprendimientos multifuncionales (Seuneke y Bock, 2015). Sin embargo, ante la persistencia de los roles de género, es necesario ampliar la mirada. Brandth y Haugen (2010) explican que, la diferenciación entre el trabajo agrícola y el turístico están influenciados por el binomio interior-exterior y la estructura tradicional del reparto de tareas por género. De acuerdo con las autoras, esta dicotomía juega un papel decisivo para la definición de nuevas identidades en los roles de la familia y del emprendimiento turístico.

La distinción que proponen Brandth y Haugen (2010) permite trasladar el análisis a nivel de territorio y destino, articulado por el doble papel que juega la familia rural. Ya que las mujeres habitualmente integran su trabajo doméstico con la empresa turística. Con ello es posible analizar el trabajo invisible de las mujeres para sostener el territorio a nivel privado y el destino a nivel público. Además, bajo la línea de la teoría ecofeminista se amplía el análisis a todos los procesos necesarios para sostener la vida (Pérez, 2014). En esta investigación la mujer desarrolla su vida en tres niveles: la familia, el territorio y el destino. Los trabajos que realiza en cada ámbito no son igualmente reconocidos, ni económica ni socialmente (Herrero, 2015). De tal manera que permite analizar las estructuras de valores necesarias para el funcionamiento de los destinos turísticos.

Sobre el destino turístico rural la información es limitada, pues fundamentalmente se orienta a su posicionamiento o sostenibilidad, sin diferenciar el rol de la mujer dentro de la estructura de funcionamiento del destino turístico rural. La literatura sobre todo ha profundizado en temas sobre el alojamiento rural y la prestación de servicios (Brandth y Haugen, 2012). También hay una limitada investigación empírica sobre las formas en que la mujer utiliza variantes como el agroturismo para dar a conocer la cultura rural (Wright y Annes, 2014).

Sin embargo, en el ámbito del emprendimiento, Annes y Wright (2015) identificaron en contextos franceses la dependencia de la actividad turística de la aprobación del hombre. Esta no debe interferir con las tareas de cuidado ni las tareas agrícolas asignadas a las mujeres. Navarro et al. (2008) explican que, en contextos españoles, la actividad turística, además, debe contribuir al sostenimiento financiero de la economía familiar. Con todo, se ha reconocido que la mujer protagoniza la experiencia turística, negocia el diseño del producto turístico con los agricultores, genera redes de cooperación (Brandth y Haugen, 2012) y por ello es relevante analizar los roles de género que persisten y de los cuales se beneficia el destino. 


\section{METODOLOGÍA}

La línea de investigación de este artículo se centra en el protagonismo de la mujer como pilar para el desarrollo territorial y la mejora de los procesos de construcción del destino turístico. El objetivo de la investigación es analizar la implicación de la mujer en la actividad turística y profundizar en la importancia del trabajo invisible para el sostenimiento de los destinos turísticos. Al mismo tiempo trata de identificar las condiciones específicas que son necesarias para el sostenimiento de las tres dimensiones en las que está inmersa la mujer: la familia, el territorio y el destino. La investigación realizada se focaliza en la experiencia de los territorios LEADER de la Región de Murcia. Para nutrir el análisis, se apoya de las conclusiones que emergieron de la observación de zonas vulnerables de la España rural, de México, con objeto de contar con una óptica latinoamericana, y de Tailandia, para una perspectiva propia del Sudeste Asiático. De este modo, se dota de profundidad al análisis de las dinámicas locales en un entorno globalizado.

Es un estudio cualitativo inductivo que se realizó a partir de la metodología de la Teoría Fundamentada (TF) de tipo glaseriano. Glaser (1992) la define como "una metodología de análisis unida a la recogida de datos que utiliza un conjunto de métodos, sistemáticamente aplicados para generar una teoría inductiva [...] o un conjunto integrado de hipótesis conceptuales, sobre el área sustantiva que es objeto de estudio" (p. 30). Con este enfoque de investigación es posible analizar contextos locales a partir de la mirada de los agentes del territorio. Mediante el uso de herramientas para producción y recolección de datos, se construye un diálogo entre los actores y el investigador, que facilitan la comprensión de las singularidades del objeto de estudio.

Charmaz (2006) distingue cinco elementos característicos de la TF: la emergencia, el análisis en espiral, el método comparativo constante (MCC), el muestreo teórico y la saturación teórica. La emergencia es uno de los aspectos más importantes de la corriente glaseriana pues permite confiar en lo que se observa, de manera que, a medida que se realiza el análisis, se puede ver surgir las respuestas a lo que está sucediendo en la realidad. Este proceso conlleva una elevada incertidumbre, pero da flexibilidad al investigador para tomar decisiones en cada momento de la investigación. El análisis en espiral es un proceso simultáneo de recogida y análisis de los datos, lo cual facilita no perder el control en la investigación y reduce la incertidumbre, porque es posible ver lo que se obtiene de los datos. El MCC sirve para analizar los datos en tres niveles (abierto, selectivo y teórico). El muestreo teórico es la herramienta para la construcción de la muestra por saturación teórica. Es decir, es la búsqueda de información en términos de relevancia y no en función del número de personas, siendo la saturación teórica la que indica el fin del trabajo de campo.

A diferencia de otro tipo de metodologías que realizan una descripción de la realidad, la TF se focaliza en la construcción de proposiciones teóricas para explicar procesos. De ahí su utilidad para explicar el papel de la mujer rural en los destinos turísticos. Mediante la "familia de las seis C" (Carrero et al., 2012, p.40), se identifica, primero, las causas, contextos y condiciones de conciliación de las dinámicas de la mujer en la esfera privada de la familia y la esfera pública donde se realiza la actividad turística. Además, con la "familia de procesos" (Carrero et al., 2012, p.41) se ha realizado la búsqueda de modelos causales o de condición y de progresiones, transiciones o ciclos para profundizar en la investigación del trabajo de la mujer rural en el territorio en sentido amplio, indagando en aquello que posibilita su transición a destino. Con la metodología de la TF no se parte 
de hipótesis preconcebidas, por lo que las conclusiones y principales aportaciones de este artículo han sido resultado del triple proceso de codificación -abierto, selectivo y teóricoguiado por el modelo concepto indicador. Las conclusiones se articulan a partir de la realidad de la Región de Murcia reforzadas con aspectos relevantes aportados por los casos complementarios español, tailandés y mexicano.

\section{DESARROLLO DE LA INVESTIGACIÓN}

El trabajo de campo se realizó en territorios LEADER de la Región de Murcia como eje principal. Se eligió este lugar dado el elevado número de expedientes LEADER promovidos por mujeres en la zona. La media en la Región se sitúa en el 43,4 \%, con más de ocho puntos por encima de la media nacional (34,68 \%) (Martínez et al., 2007). Además, principalmente son sobre turismo rural (García Marín, 2011; Nieto y Cárdenas, 2017). Los casos complementarios de otros contextos de la España rural responden a la aplicación del muestreo teórico. Es decir, fueron experiencias mencionadas por las propias entrevistadas, que por su experiencia de trabajo aportaron información relevante a la investigación. Así se integró un caso de Ahigal de los Aceiteros y uno de Aldeadávila de la Ribera, ambos en Salamanca.

Por otro lado, para profundizar en el análisis de las dinámicas femeninas, fue incorporada la experiencia de mujeres del municipio de Cuetzalan en Puebla, México. Este país fue elegido por ser el destino más visitado en América Latina. De acuerdo con datos de la OMT, se ubica en el séptimo lugar por número de turistas internacionales y el sector turístico aporta un 17,2 \% al PIB nacional (OMT, 2019). Concretamente, Puebla destaca entre los siete Estados con mayor oferta de proyectos ecoturísticos y de turismo rural. Cuetzalan es uno de los municipios pioneros del turismo rural en México, el cual es financiado por el gobierno federal a través del programa Pueblos Mágicos (Juárez, Benito y Galindo, 2010).

Finalmente, se incorpora la experiencia de Tailandia que, al igual que México, es el destino que recibe el mayor número de turistas de su ámbito geográfico, situándose entre los diez países más visitados del mundo (OMT, 2019). Concretamente en Chanthaburi, se localiza el Real Centro de Estudios de la bahía de Kung Krabaen, uno de los proyectos desarrollados en la década de los ochenta para mejora del entorno natural y de la calidad de vida de la población. Fue creado bajo la Filosofía de la Sufficiency Economy y sirve de modelo base para la implementación de proyectos agroturísticos en la zona. Además, forma parte del Programa de Viajes de Agroturismo de la Autoridad Turística de Tailandia (Office Of The Royal Development Projects Board, 2017).

En todos los casos se trata de contextos rurales con experiencias de turismo impulsadas por mujeres y promovidas por programas públicos. Se tuvieron en cuenta tres perfiles de mujeres: gestoras de programas o proyectos, emprendedoras y trabajadoras del sector turístico. Las herramientas para producción de datos fueron la entrevista en profundidad y la observación participante. Las entrevistas se realizaron en cinco etapas de mayo 2017 a mayo 2019 ${ }^{1}$ : primero en la Región de Murcia, posteriormente en los contextos de la

\footnotetext{
${ }^{1}$ En la teoría fundamentada, la recogida de datos, la codificación y el análisis se realiza de forma simultánea, y solo se puede iniciar una nueva entrevista tras haber concluido el análisis de la entrevista previa. Por ello el trabajo de campo abarca un horizonte temporal amplio.
} 
España rural, México, Tailandia y, en último lugar, para el cierre del trabajo de campo, nuevamente la Región de Murcia (Figura 1).

Figura 1. Trabajo de campo y recogida de información

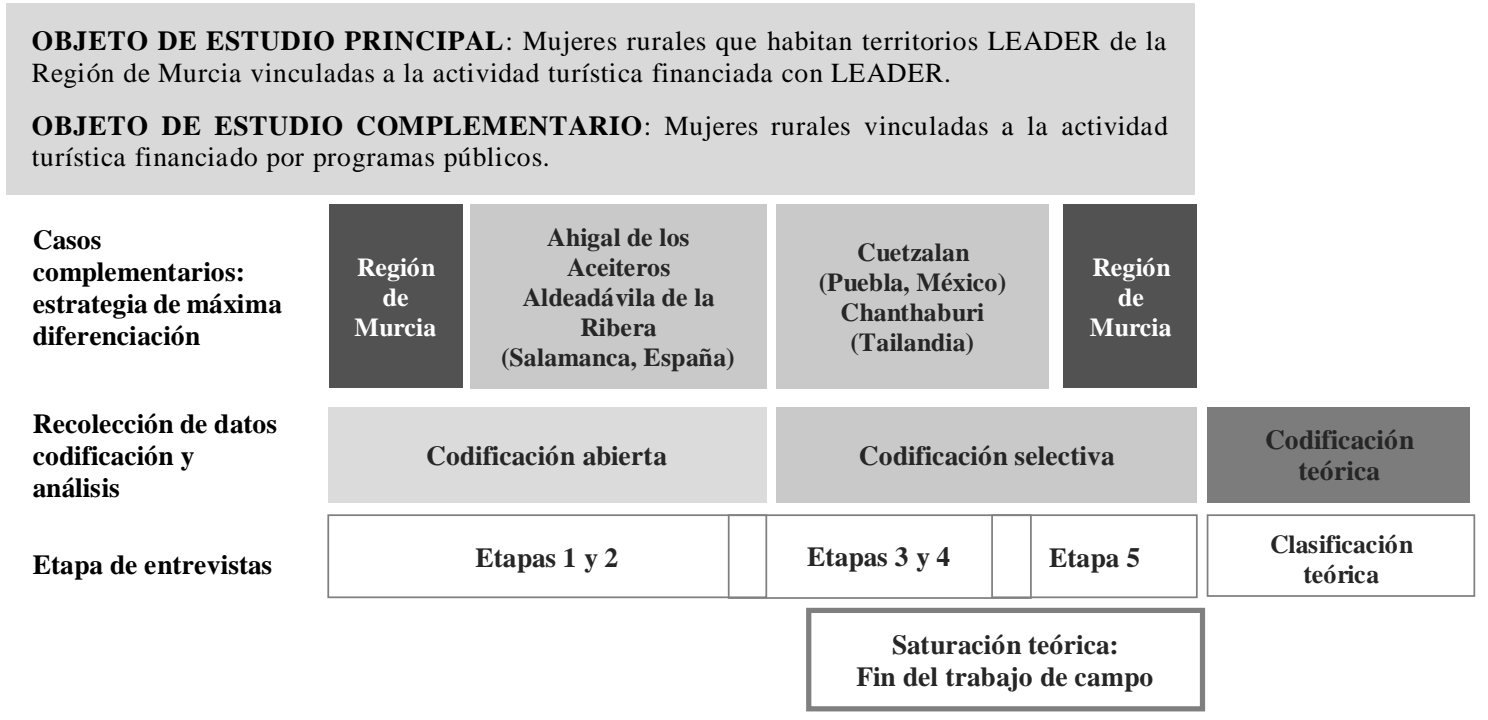

Fuente: elaboración propia con información de diario de campo.

La selección de las primeras tres informantes se realizó por muestreo teórico, es decir, por la relevancia de la información que podía aportar. Las sucesivas informantes se eligieron utilizando, además del muestreo teórico, la estrategia de bola de nieve. La entrevista contó con un guion, que, a medida que se realizaba el análisis, incorporaba nuevas variables a considerar. La duración de cada una de las entrevistas estuvo en el rango de los 45-90 minutos. Se obtuvo una muestra por saturación teórica de 17 entrevistas, con un total de 12 informantes (Figura 2). Un hecho interesante es que siete de las 12 informantes cumplían más de un rol en la en la actividad turística, especialmente entre las emprendedoras.

Figura 2. Conformación de la muestra
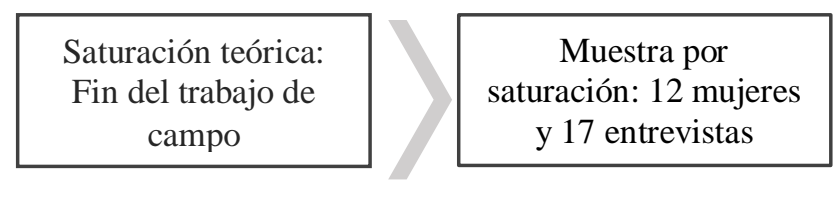

\section{3 perfiles:}

- Gestoras de programas o proyectos

- Emprendedoras

- Trabajadoras del sector turístico

Fuente: elaboración propia con información de diario de campo.

La codificación abierta concluyó con un total de 56 códigos sobre ocho temáticas: las dinámicas de la mujer rural, el cuidado de la familia, el cuidado del territorio, la dinámica de la actividad económica, la diversificación del territorio, la situación de la actividad turística, la gestión del destino y las políticas públicas e instrumentos de financiación. Durante la codificación selectiva se inició el proceso de la búsqueda de la categoría 
central, para ello se ajustaron los datos a 42 códigos sustantivos en seis familias: dinámica de la mujer rural, el trabajo y los roles de género, dinámica general de la actividad económica, diversificación del territorio, la actividad turística y la gestión del destino y la mujer en el marco normativo y estratégico (Tabla 1).

Tabla 1. Codificación selectiva por familias

\begin{tabular}{|c|c|}
\hline $\begin{array}{l}\text { 1. Dinámica de la mujer rural } \\
\text { 1.1. ¿Qué hago? } \\
\text { 1.2. ¿Quién soy? } \\
\text { 1.3. Formación educativa } \\
\text { 1.4. El trabajo } \\
\text { 1.5. Identidad } \\
\text { 1.6. Espacios de participación }\end{array}$ & $\begin{array}{l}\text { 2. El trabajo y los roles de género } \\
\text { 2.1. El cuidado de la familia y personas } \\
\text { dependientes } \\
\text { 2.2. Los valores tradicionales } \\
\text { 2.3. La organización familiar } \\
\text { 2.4. El ingreso familiar } \\
\text { 2.5. El trabajo invisible } \\
\text { 2.6. Cuidado del territorio }\end{array}$ \\
\hline $\begin{array}{l}\text { 3. Dinámica general de la actividad } \\
\text { económica } \\
\text { 3.1. Actividades productivas tradicionales } \\
\text { 3.2. El mercado de trabajo } \\
\text { 3.3. El trabajo como complemento } \\
\text { 3.4. Nuevas cualificaciones } \\
\text { 3.5. Identidad territorial } \\
\text { 3.6. Emprendimiento }\end{array}$ & $\begin{array}{l}\text { 4. La diversificación del territorio } \\
\text { 4.1. Equilibrio entre conservación y } \\
\text { desarrollo } \\
\text { 4.2. Las diferencias del territorio } \\
\text { 4.3. Complementariedad de actividades } \\
\text { productivas } \\
\text { 4.4. Adaptación de nuevas actividades } \\
\text { 4.5. Titularidad de la tierra } \\
\text { 4.6. Desagrarización como oportunidad } \\
\text { 4.7. Innovación social } \\
\text { 4.8. Dinamización del territorio }\end{array}$ \\
\hline $\begin{array}{l}\text { 5. La actividad turística y gestión del destino } \\
\text { 5.1. Fortalezas del destino } \\
\text { 5.2. Debilidades del destino } \\
\text { 5.3. Infraestructura turística } \\
\text { 5.4. La promoción del destino } \\
\text { 5.5. La mujer en la promoción del destino } \\
\text { 5.6. Lo que pide el turista } \\
\text { 5.7. Identidad del destino } \\
\text { 5.8. Estructura de gobernanza } \\
\text { 5.9. Participación en la planificación del } \\
\text { destino }\end{array}$ & $\begin{array}{l}\text { 6. La mujer rural en el marco normativo y } \\
\text { estratégico } \\
\text { 6.1. La mujer en la agenda política } \\
\text { 6.2. Subvenciones y ayudas a la mujer } \\
\text { 6.3. Subvenciones al turismo } \\
\text { 6.4. Espacios de participación } \\
\text { 6.5. Percepción de las ayudas y subvenciones } \\
\text { 6.6. Acceso a espacios de decisión }\end{array}$ \\
\hline
\end{tabular}

Fuente: elaboración propia al finalizar el proceso de codificación selectiva.

Durante el proceso de codificación teórica se trabajó en la generación de relaciones entre los códigos y sus propiedades, se identificaron los procesos sociales básicos y con ello se obtuvo la categoría central. En la Tabla 2 se ilustra el proceso.

Tras el proceso de codificación teórica se inició el trabajo de clasificación teórica. Esta fase estructura el marco explicativo del informe final. Para ello la información se ordenó en torno a tres familias conceptuales: 1) la situación general de la mujer, donde se integran aspectos como la formación, los roles de género y los aspectos estructurales del medio rural; 2) la mujer en el territorio, donde se integra el trabajo que realiza para sostener la vida en el medio rural y en torno a la actividad turística; y 3) los aspectos vinculados a la mujer en el destino, donde se distingue su rol en la esfera pública (Figura 3). 
Tabla 2. Codificación teórica

\begin{tabular}{|c|c|c|c|}
\hline Categorías Sustantivas & Categorías Conceptuales & $\begin{array}{l}\text { Proceso Social } \\
\text { Básico }\end{array}$ & Categoría Central \\
\hline $\begin{array}{l}\text { 1. El trabajo invisible } \\
\text { 1.1. Cuidado de la familia y/o } \\
\text { personas dependientes } \\
\text { 1.2. Conservación y trasmisión } \\
\text { de cultura } \\
\text { 1.3. Cuidado del patrimonio } \\
\text { natural }\end{array}$ & $\begin{array}{l}\text { Persistencia de los roles de } \\
\text { género }\end{array}$ & \multirow[t]{2}{*}{$\begin{array}{l}\text { Resistiendo } \\
\text { (Resistir) }\end{array}$} & \multirow[t]{6}{*}{$\begin{array}{l}\text { Moldeamiento } \\
\text { La adecuación de } \\
\text { los roles de género }\end{array}$} \\
\hline $\begin{array}{l}\text { 2. Actividad económica } \\
\text { 2.1. El trabajo para } \\
\text { complemento de ingreso } \\
\text { familiar } \\
\text { 2.2. Cualificaciones }\end{array}$ & $\begin{array}{l}\text { Barreras de acceso a } \\
\text { puestos especializados }\end{array}$ & & \\
\hline $\begin{array}{l}\text { 3. Punto de inflexión entre las } \\
\text { dinámicas de la familia y del } \\
\text { destino } \\
\text { 3.1. Desagrarización como } \\
\text { oportunidad } \\
\text { 3.2. Innovación } \\
\text { 3.3. Incorporación de la } \\
\text { actividad turística } \\
\text { 3.4 Convivencia y destino }\end{array}$ & $\begin{array}{l}\text { La multifuncionalidad del } \\
\text { territorio }\end{array}$ & $\begin{array}{l}\text { Negociando } \\
\text { (Negociar) }\end{array}$ & \\
\hline $\begin{array}{l}\text { 4. De la Familia al territorio } \\
\text { 4.1 La adopción de la actividad } \\
\text { 4.2. Estructura de gestión } \\
\text { familiar } \\
\text { 4.3. Red de cooperación }\end{array}$ & $\begin{array}{l}\text { Armonización de las } \\
\text { actividades }\end{array}$ & \multirow[t]{3}{*}{ Moldeamiento } & \\
\hline $\begin{array}{l}\text { 5. Del territorio al destino } \\
\text { 5.1. Los valores del destino } \\
\text { 5.2. Diversificación de las } \\
\text { funciones productivas del } \\
\text { territorio } \\
\text { 5.3. La cohesión territorial }\end{array}$ & $\begin{array}{l}\text { El moldeamiento de los } \\
\text { roles de género para la } \\
\text { experiencia turística }\end{array}$ & & \\
\hline $\begin{array}{l}\text { 6. Rol y expectativas } \\
\text { 6.1. Redefinición de actividades } \\
\text { 6.2. Valoración interna-externa }\end{array}$ & $\begin{array}{l}\text { Reequilibrio de la } \\
\text { actividad productiva }\end{array}$ & & \\
\hline
\end{tabular}

Fuente: elaboración propia durante el proceso de codificación teórica.

El resultado de la clasificación teórica tuvo como fin generar un informe ordenado de las dinámicas de la mujer rural entre la esfera privada de la familia y la esfera pública del destino turístico. El marco explicativo se centra en la experiencia de la Región de Murcia, pero se refuerza con datos puntuales de los contextos complementarios. Como tal, no presenta la situación en particular de un solo grupo de mujeres, sino que ofrece una explicación que permite contar con una visión genérica desde la perspectiva de las mujeres. Así, el informe final es estructurado en tres etapas:

Momento 1. La esfera privada de la familia: resistiendo

- El trabajo invisible para sostener la familia: persistencia de los roles de género. 
- El trabajo remunerado como complemento del ingreso familiar: barreras de acceso a puestos especializados, cualificación de la mujer.

- El trabajo de cuidado del territorio: conservación y trasmisión de valores, cuidado del patrimonio natural.

Figura 3. Dinámicas de la mujer rural

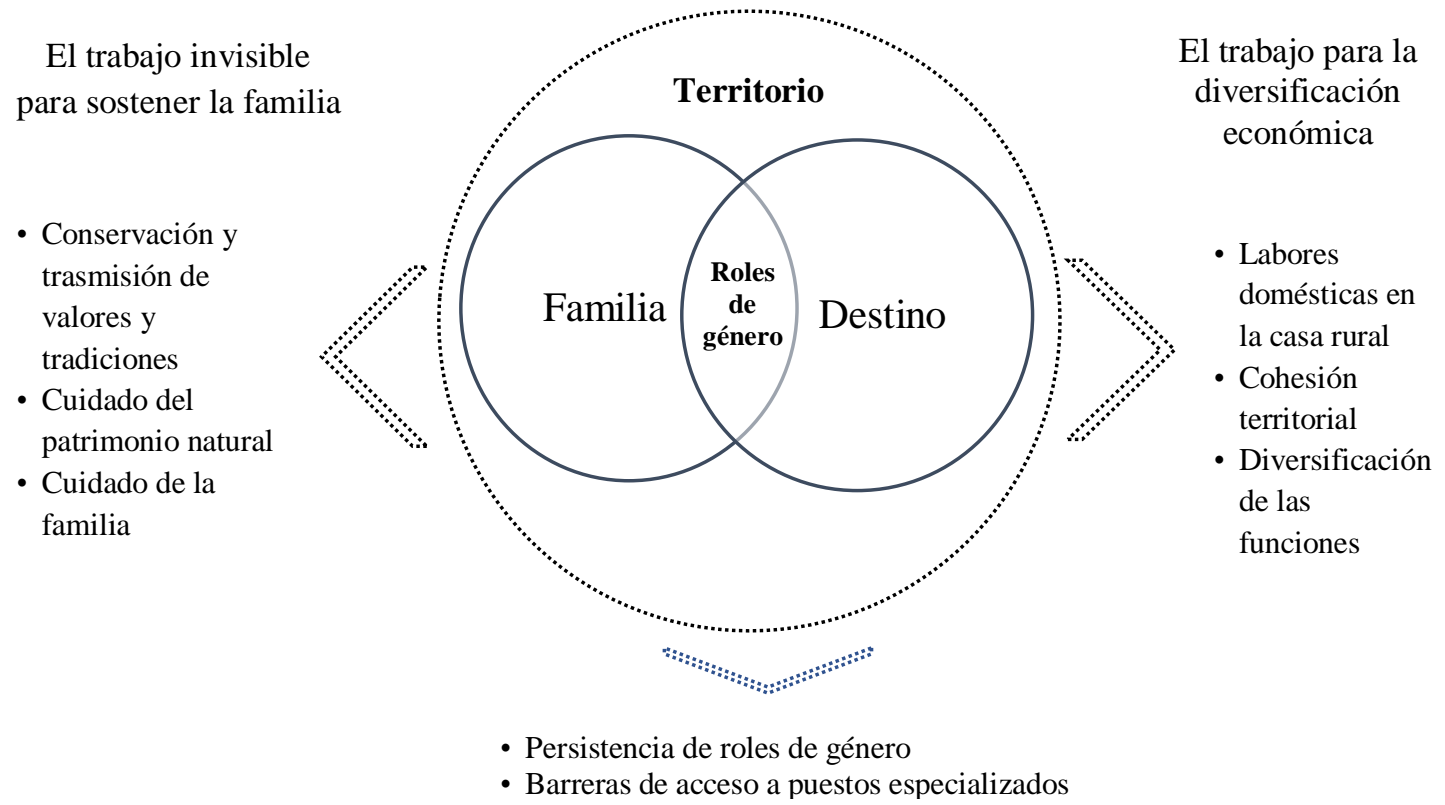

Fuente: elaboración propia durante el proceso de clasificación teórica.

Momento 2. La transición de los roles tradicionales del hogar al destino: negociando

- Multifuncionalidad del territorio: la desagrarización como oportunidad, la incorporación de la actividad turística.

- Armonización de las actividades: La adopción de la actividad turística, estructura de gestión familiar y red de cooperación.

Momento 3. De la esfera pública del destino a un ecosistema de servicios: moldeamiento

- Diversificación de las funciones productivas del territorio: la identidad del destino, la cohesión territorial.

- Reequilibrio de la actividad productiva: Redefinición de actividades. Valoración interna-externa.

\section{LA MUJER RURAL EN EL DESTINO TURÍSTICO}

Para que el proceso de moldeamiento tenga origen, se identifican un conjunto de transacciones que tienen como resultado el ajuste entre la esfera pública y la privada. En este apartado se presenta el desarrollo de cada una de las etapas del marco explicativo el 
cual describe el rol de la mujer en el destino turístico y su proceso de adecuación de los roles de género para el soporte del destino.

El primer momento de ajuste para el proceso de moldeamiento es generado por la transición de la esfera privada. Por un lado, muestra a la mujer en núcleo familiar y el punto de fuga para su desinvisibilización (Wright y Annes, 2014). Partiendo de los rasgos generales del trabajo invisible que realiza la mujer (Pérez, 2014), el trabajo doméstico sigue siendo poco valorado y continúa la división tradicional de tareas en el núcleo familiar (Shortall et al., 2020). Cuestiones como la masculinización de los sectores productivos del sector primario y la titularidad y acceso a la tierra se mantienen y limitan el acceso de la mujer a las actividades productivas fuera del hogar. "Hay señoras que su marido se dedicaba a echar jornales, no tenían terreno suficiente y la mujer está en casa, pero porque no tenía posibilidad de trabajar ahi'".

Hay una notable diferencia de nivel de formación, entre las mujeres de edad más avanzada y las mujeres jóvenes, que condiciona el acceso a puestos de trabajo especializados. Es decir, en los grupos de edad más avanzada la mujer adolece de la mínima formación, siendo las jóvenes las que cuentan con un mayor nivel educativo (Baylina, 2019). Esto se traduce en el éxodo rural femenino ya documentado por Camarero y Oliva (2016). Una situación que además condiciona las limitaciones de movilidad (Camarero, 2017b): " $L a$ gente de ahí está muy parada. Hay muchas mujeres con cincuenta años, amas de casa que podrían haber optado por un trabajo y al no tener carné, al no tener posibilidad de desplazamiento, pues no están haciendo nada, están de amas de casa y nada más".

En términos de formación y acceso al empleo, un hecho constatado en las realidades observadas son algunos elementos esperanzadores de cara al futuro, por ejemplo, la aparición de nuevas oportunidades de empleo en el mundo rural y la llegada de una nueva generación de mujeres jóvenes, formadas e independientes, que desean desarrollar su proyecto de vida en el pueblo más allá de la actividad turística, pero en sincronía con esta (Sánchez-Oro y Pérez, 2007; Baylina, 2019): “'La calidad de vida es estupenda! La pena es que muchas de las personas no han aprovechado más, yo me veo con mi tierra trabajando con una empresa de ocio y tiempo libre para lo que salga".

Entre los entornos masculinizados, como es el caso español y los entornos feminizados como es el caso mexicano, solo se puede confirmar la necesaria presencia de personas para el cuidado en sentido amplio del territorio (Shiva y Mies, 2016). Sin embargo, los datos mostraron una división de opiniones respecto al valor del territorio y su cuidado. Las personas mayores tienen una forma negativa de ver el medio rural y no se implican en el cuidado de la misma manera en que lo hacen las mujeres jóvenes. Por tanto, el cuidado del territorio y el cuidado del patrimonio natural es realizado sobre todo por las mujeres jóvenes, función que se suma a su tradicional labor de trasmisión de valores: " $L o$ ecológico es algo de nuestra generación, o sea, las generaciones anteriores no lo entienden. A ver, nosotros tenemos una pequeña huerta y yo no hago más que decirle a mi tio, por favor, chacho, no lo sulfates tanto. Siempre viene con olor. 'Si el azufre no es nada, el azufre no es nada'. Y digo: 'ya, en pequeñas cantidades no es nada, pero que tu vienes todos los días oliendo a azufre'. Ellos no lo valoran, no lo entienden...".

En el segundo momento la mujer como responsable de la transmisión de saberes los transfiere y materializa en la creación de un destino turístico. Debido a la centralidad que adquiere, tanto en las políticas públicas como en la gestión de proyectos (Shortall, 2015), tiene la capacidad de generar estrategias territoriales basadas en el principio de especificidad de activos como mecanismo de diferenciación. Esto es especialmente relevante para la construcción del territorio, que requiere de la movilización de los actores 
a partir de sus recursos endógenos y su cultura (Carpio, 2002; Pecqueur, 2005; VázquezBarquero 2017). "Por eso, mi afán y todo este modelo que me he creado en mi mente, hago por mantener el castillo donde nací, donde me crie, porque es un legado de mi familia, de su pasado y sus usos y costumbres".

El punto de inflexión en la situaciones estructurales de la mujer viene dado por un proceso de negociación. El cual se inicia por la diversificación económica (Alonso y Trillo, 2014) gracias a la desagrarización del territorio (Camarero, 2017a). Este hecho aparece como un momento de oportunidad para reconvertir las funciones productivas del territorio y ampliar su multifuncionalidad (Seuneke y Bock, 2015). "Pero eso no fue fácil, fue necesario, primero convencer a la familia, los bancos, al líder del Nordeste que cogió y me ayudó. Para mí fue una herramienta bastante principal. No tengo muchos conocimientos. Entonces, si alguien te guía te va ayudando a superar esos pequeños obstáculos pues al final sale un proyecto".

Sucede por la asunción de la mujer a la titularidad de las explotaciones agrarias tras un proceso de relevo generacional (Cruces y Palenzuela, 2006) y la incorporación de nuevas actividades para el desarrollo profesional. "Nací y crecí en un entorno vitivinícola, rural por supuesto, mi familia siempre ha sido agricultora, sin embargo y como todas éramos chicas, cómo nos íbamos a dedicar al campo. Eso en mi padre era como algo un poco asi'”.

Así, se incorpora la actividad turística como sector compatible y complementario a la agricultura (Brandth y Haugen, 2012). Simultáneamente se da la identificación de nuevas funciones vitales del territorio, compensando la pérdida de peso del sector agrícola y ganadero (Camarero, 2017a; Seuneke y Bock, 2015). Esto es especialmente visible en contextos como el medio rural español y mexicano -e, incipientemente, también en el sudeste asiático- donde la presencia de la mujer combina el turismo como parte del cuidado del territorio, del sostenimiento de la economía familiar (Brandth y Haugen, 2007; Navarro et al., 2008) y de la conservación del paisaje "Me embarqué en un modelo de casa rural, en un proyecto donde la diversificación de ese modelo agrario me ha llevado a tener una casa rural y explotarla de forma turística". En este nivel de gestión, el territorio deja de ser un espacio pasivo para configurarse como un territorio vivo de manera que no se limita a recibir turistas, sino que avanza la vertebración del resto de actividades, emergiendo propuestas como el oleoturismo, el agroturismo, el enogastroturismo, etc.

La adopción de la actividad turística conlleva una armonización entre las actividades de la esfera privada y las nuevas actividades para la construcción del destino (Annes y Wright, 2015). El trabajo de negociación de la mujer sirve de puente para la creación de una red de cooperación. Cuando el desarrollo de la actividad turística parte de la creación de un territorio, entendido este como la movilización de los actores en torno a una estrategia territorial ${ }^{2}$ (Pecqueur, 2005; Ramos y Garrido, 2011), facilita la complementariedad de las acciones y el trabajo en red (Brandth y Haugen, 2012). "Hasta que no nos reunimos con Bienve, con gente de la Paca, con su sabiduría y su experiencia fue la que nos encauza a decir es esto lo que necesitamos. Desde el punto de vista asociativo, [...] es un ejemplo de cómo se debe resolver. Aun así, hay muchos ingenuos que no lo creen, porque siempre se ha creado rivalidad entre ellos. Lo difícil es ahora unirse, porque siempre hemos estado separados, o sea, que no es tarea fácil. Ahí somos

\footnotetext{
${ }^{2}$ En territorios LEADER este hecho se realiza al margen de las estrategias de desarrollo participado ya que este instrumento no suele facilitar la cooperación por la estructura de los Grupos de Acción Local (Thuesen, 2010).
} 
30 por lo menos, ahí hay muchísima población. Lo que debemos es seducir al resto, porque hay miedo. Entonces, ahora tenemos la labor de unirnos y de demostrar que esto va a ser beneficioso para todos".

Se coincide con Anns y Wright (2015), que el empoderamiento de la mujer rural no ocurre por el turismo en sí mismo. De acuerdo con los datos de esta investigación es producto del proceso de negociación entre la familia y la mujer para adoptar el turismo como actividad económica complementaria. En este nivel se puede hallar una diferencia entre quienes tienen un emprendimiento o participan en algún nivel de gestión. En ellas la redefinición de actividades es más significativo pues les permite redefinir su identidad y ganar confianza". "Ahora digo que soy una autónoma gerente de una explotación agroturística. Esa es mi definición. Una persona normal y corriente, pero al final tengo un modo de vida".

El tercer momento, caracterizado por la transición desde el destino a un ecosistema de servicios, está influenciado por la reproducción de los estereotipos de género asentados en el medio rural en los que la mujer está al servicio la familia (Sparrer, 2003). Si bien es cierto que las actividades que realiza la mujer en el destino reproducen las actividades tradicionales del hogar (Brandth y Haugen, 2010) también lo es que, cuando es el fruto de un proceso de transacción no es percibido como un problema. En este sentido, en los contextos donde no se presenta un enfoque integrado de la gestión del territorio, el destino turístico únicamente se asume como un soporte de productos turísticos y no como el instrumento de revitalización del territorio (Camarero y Oliva, 2013). Es decir, se reduce a la promoción de un servicio turístico de descanso sin ninguna trascendencia en la dinámica del territorio y, por tanto, en las dinámicas de la mujer. Este hecho contribuye a que se produzca una diversificación de las funciones productivas del territorio y un reequilibrio de sus actividades productivas (Brandth y Haugen, 2007; Anns y Wright, 2015). De forma que, los destinos donde se tiene una gestión participada y ascendente, el papel de la mujer se percibe como un elemento que facilita el diseño de actuaciones transversales y la persistencia de los roles de género se suaviza.

El proceso de moldeamiento se produce en paralelo a este proceso de diversificación de las acciones productivas. Por ello mediante un enfoque territorial, que parte de la construcción del territorio, se alcanza el reequilibrio de las actividades productivas del mismo. Un equilibro que compagina la valoración externa a nivel del destino turístico con las dinámicas internas a nivel de la familia (Brandth y Haugen, 2010) que a su vez avanza en la redefinición de actividades del territorio (Berdulay, 2009): "mi actividad, bueno, la llevan ... yo soy una mujer. Con el carácter te tienes que pulir porque si no... No te puedes dejar. Me busco mucho la vida, no me conformo con quedarme ahí y solo ordeñar unas ovejas. Creo que hay que hacer más cosas [...] Estos son mis aceites ecológicos. Y yo lo que les ofrecí fue hacer algo diferente, una ruta ornitológica, una visita a mi almazara y catas de aceite. Otra cosa no tengo, pero seguridad... Mucha". Finalmente, la definición de la identidad del destino se realiza a partir de la estrategia territorial, ya que en ella se hallan las especificidades del territorio al abrigo de una marca territorial (Ramos y Garrido, 2011).

\footnotetext{
${ }^{3}$ Este hallazgo, contribuye con los estudios que ven lo rural como parte integral de la construcción real de identidades de género, así como las formas en que se acentúan y se negocian (Little y Panelli, 2003).
} 


\section{CONCLUSIONES}

La mujer es esencial para conseguir territorios rurales exitosos, pero para que esto suceda es necesario que se produzca un moldeamiento. En ese moldeamiento, la existencia de canales de participación que posibiliten el desarrollo bottom-up es determinante (Derkzen y Bock, 2007; Thuesen, 2010). Además, este proceso de moldeamiento contribuye al soporte del destino turístico. El territorio se convierte en el lugar donde conviven las dinámicas de la mujer de la esfera privada y la esfera pública. Este plano actúa como pilar del destino y del empoderamiento de la mujer, ya que articula una red de cooperación entre otros agentes del territorio para generar una estrategia territorial para el destino. El empoderamiento de la mujer no viene dado por la actividad turística, sino por el proceso de negociación para la incorporación de la actividad turísticas. Es decir, pasa de un momento de aceptación, que se denominó resistencia en la esfera privada, a uno de amoldamiento para la esfera pública. Además, el nivel de confianza dependerá del nivel de implicación de la mujer en la esfera pública, ya que ganan más confianza las mujeres emprendedoras o gestoras de programas respecto de las trabajadoras del sector turístico.

Las funciones, competencias y conductas que desarrolla la mujer son similares en la esfera pública y en la esfera privada. Mientras que en la primera contribuye a la cohesión del territorio y el destino, en la segunda contribuye a ampliar la frontera multifuncional. Las acciones que realiza la mujer en el núcleo familiar suelen ser similares en la actividad turística. En la esfera pública se lleva a cabo la actividad turística, en la cual la mujer tiene la posibilidad de armonizar tanto las actividades de la familia como las actividades para los turistas. La mujer rural, a pesar de ampliar la frontera de la multifuncionalidad del territorio, opta por mantener las estructuras tradicionales de convivencia ya que esto contribuye a la cohesión del territorio y del destino. No obstante, cuando es producto de una negociación, esto no se manifiesta como un problema.

En futuras investigaciones, sería interesante profundizar en el proceso de negociación de la mujer, ya que podría ampliar el conocimiento sobre los factores que favorecen la cohesión social del territorio y el capital social necesario para el posicionamiento de un destino turístico. En esa línea, los estudios sobre ruralidad e identidad de Little (2002, 2015), Little y Panelli (2003), Seuneke y Bock (2015) y Brandth, (2002, 2012) son de interés para aplicar al estudio de la gestión de los destinos. Ya que, el capital social generado por las mujeres aparece como un elemento relevante, pero, solo en el caso español y mexicano ocupa un lugar central. Ambos contextos muestran la creación de estructuras de gestión lideradas por mujeres que funcionan tanto para el territorio y como para el destino, por tanto, son susceptibles de análisis. También sería de interés profundizar en estudios cualitativos sobre conservación y mantenimiento de valores, diversidad cultural, etnográfica y protección del medio natural, ya que en esta investigación solo emergieron algunos datos sobre el contexto mexicano y forma parte de la construcción de la identidad de las mujeres (Little, 2015) y de los valores de los destinos.

\section{REFERENCIAS}

Alburquerque, F. (2001). Ajuste estructural e iniciativa de desarrollo local. Banco Nacional de Comercio Exterior, 675-682. Recuperado de: http://revistas.bancomext.gob.mx/rce/magazines/33/1/RCE.pdf (16/06/2018). 
Alonso, N., y Trillo, D. (2014). Women, Rural Environment and Entrepreneurship. Procedia - Social and Behavioral Sciences, (161), 149-155. https://doi.org/10.1016/j.sbspro.2014.12.039.

Annes, A., y Wright, W. (2015). Creating a room of one's own: French farm women, agritourism and the pursuit of empowerment. Women's Studies International Forum, (53), 1-11. https://doi.org/10.1016/j.wsif.2015.08.002.

Baylina, M (2019). La mujer como eje vertebrador de la nueva ruralidad. Un estado de la cuestión. En E. Cejudo, y F. Navarro (eds.), Despoblación y transformaciones sociodemográficas de los territorios rurales: los casos de España, Italia y Francia (pp. 153-176). Università del Salento. https://doi.org/10.1285/i26113775n3p153.

Berdoulay, V. (2009). La historia de la geografía en el desafío de la prospectiva. Economía y Sociedad: Esbozo de Sociología Comprehensiva, (51), 9-23.

Blasco, D., Guia, J., y Prats, L. (2013). Tourism destination zoning in mountain regions: A consumer-based approach. Tourism Geographies, 16(3), 512-528. https://doi.org/10.1080/14616688.2013.851267.

Brandth, B. (2002). Gender Identity in European Family Farming: A Literature Review. Sociologia Ruralis, 42(3), 181-200. https://doi.org/10.1111/1467-9523.00210.

Brandth, B., y Haugen, M. S. (2007). Gendered work in family farm tourism. Journal of Comparative Family Studies, 38(3), 379-393. https://doi.org/10.3138/jcfs.38.3.379.

Brandth, B., y Haugen, M. S. (2010). Doing farm tourism: The intertwining practices of gender and work. Signs, 35(2), 425-446. https://doi.org/10.1086/605480.

Brandth, B., y Haugen, M. S. (2012). Farm tourism and dilemmas of commercial activity in the home. Hospitality and Society, 2(2), 179-196. https://doi.org/10.1386/hosp.2.2.179_1.

Brandth, B., y Haugen, M. S. (2014). Embodying the Rural Idyll in Farm Tourist Hosting. Scandinavian Journal of Hospitality and Tourism, 14(2), 101-115. https://doi.org/10.1080/15022250.2014.899136.

Camarero L., Sampedro R. (2016). Exploring female over-migration in rural Spain. Employment, care giving and mobility. In K. Wiest (ed.), Women and migration in rural Europe. Labour markets, representations and policies (pp. 189-208). Ashgate.

Camarero, L. (2017a). Trabajadores del campo y familias de la tierra. Instantáneas de la desagrarización. Ager. Revista de Estudios sobre Despoblación y Desarrollo Rural (23), 163-195. https://doi.org/10.4422/ager.2017.01.

Camarero, L. (2017b). Por los senderos de la despoblación rural: notas desde la diversidad social. Documentación social, (185), 19-35. Recuperado de: https://www2.uned.es/dpto-sociologia-

I/departamento_sociologia/luis_camarero/DS.pdf (17/04/2020).

Camarero, L. (Coord.) (2009). La Población Rural en España. De los desequilibrios a la sostenibilidad social. La Caixa. Recuperado de: https://obrasociallacaixa.org/documents/10280/240906/vol27_es.pdf/2b8d7898137d-4927-bea0-8cfef7c6f4f8 (22/01/2020).

Camarero, L., y Oliva, J. (2013). Touristic Processes, Generic Rurality and Local Development. In E. Figueiredo, and A. Raschi (ed.), Fertile Links? Connections 
between tourism activities, socioeconomic contexts and local development in European rural areas (pp. 19-42). Firenze University Press.

Cánoves, G., Villarino, M., Blanco-Romero, A., De Uña, E., y Espejo, C. (2014). Turismo de interior: renovarse o morir. Estrategias y productos en Catalunya, Galicia y Murcia. Universitat de València Servei de Publicacions.

Carpio, J. (2000). Desarrollo local para un nuevo desarrollo rural. Anales de geografía de la Universidad Complutense, (20), 85-100. Recuperado de: https://revistas.ucm.es/index.php/AGUC/article/view/AGUC0000110085A (17/04/2020).

Carrero, V., Soriano-Miras, R., y Trinidad, A. (2012). Teoría Fundamentada Grounded Theory. El desarrollo de teoría desde la generalización conceptual. Cuadernos Metodológicos, (37), CIS.

Cruces, C., y Palenzuela, P. (2006). Emprendedoras rurales en Andalucía: posibilidades y límites de sus estrategias. Revista española de estudios agrosociales y pesqueros, (211), 239-306. Recuperado de: https://www.mapa.gob.es/app/publicaciones/art_datos.asp?articuloid=1202\&codre vista=REEAP (18/10/2018).

Charmaz, K. (2006). Constructing Grounded Theory. SAGE Publications.

Derkzen, P., y Bock, B. B. (2007). The construction of professional identity: Symbolic power in rural partnerships in the Netherlands. Sociologia Ruralis, 47(3), 189-204. https://doi.org/10.1111/j.1467-9523.2007.00440.x.

Dessein, J., Bock, B. B., y de Krom, M. (2013). Investigating the limits of multifunctional agriculture as the dominant frame for Green Care in agriculture in Flanders and the Netherlands. Journal of Rural Studies, (32), 50-59. https://doi.org/10.1016/j.jrurstud.2013.04.011.

Dredge, D. (1999). Destination place planning and design. Annals of Tourism Research 26(4), 772-79. https://doi.org/10.1016/S0160-7383(99)00007-9

García Marín, R. (2011). Turismo y desarrollo rural en la Comarca del Noroeste de la Región de Murcia: Los programas europeos LEADER. Cuadernos de Turismo, (27), 419-435. Recuperado de: https://revistas.um.es/turismo/article/view/140031 (18/10/2018).

Garofoli, G. (1991). Desarrollo econçomico, Organización de la Producción y Territorio. In A. Vázquez-Barquero, y G. Garofoli (eds.), Desarrollo Económomico Local en Europa (pp. 53-71). Colegio de Economistas.

Glaser, B. G. (1992). Basic of Grounded Theory Analysis, Emergence vs. Forcing. Sociology Press.

Guinjoan, E., Badia, A., y Tulla, A. F. (2016). El nuevo paradigma de desarrollo rural. Reflexión teórica y reconceptualización a partir de la Rural Web. Boletín de la Asociación de Geógrafos Españoles, (71), 179-204. https://doi.org/10.21138/bage.2279.

Herrero, Y. (2015). Cambiar las gafas para cambiar el mundo. Libros en acción.

Juárez S, P., Benito, R., y Galindo, V. M. (2010). Turismo rural y desarrollo territorial en espacios indígenas de México. Investigaciones geográficas, (48), 189-208. DOI: https://doi.org/10.14198/INGEO2009.48.07. 
Linck, T. (2014). Los Siales entre Apropiación y Despojo Territorial: Hay que Darle Rumbo al Enfoque. Pampa, (6), 9-25. https://doi.org/10.14409/pampa.v1i6.3174.

Little, J. (2002). Rural geography: Rural gender identity and the performance of masculinity and femininity in the countryside. Progress in Human Geography, 26(5), 665-670. https://doi.org/10.1191/0309132502ph394pr.

Little, J. (2015). The development of feminist perspectives in rural gender studies. In B. Pini, B. Brandth, and J. Little (eds.), Feminisms and ruralities (pp. 107-118). Lexington Books.

Little, J., y Panelli, R. (2003). Gender research in rural geography. Gender, Place and Culture, 10(3), 281-289. https://doi.org/10.1080/0966369032000114046.

Martínez, E., López, N., Martín, F., Sabaté, A., y Saéz. E. (2007). Mujeres emprendedoras en el desarrollo rural. Atlas Regional de España de la participación de las mujeres rurales emprendedoras en la Iniciativa Comunitaria LEADER II. Ministerio de Asuntos Sociales, Instituto de la Mujer.

Moisy, L. (2001). Le lieu dans sa dimension touristique. Espaces et Sociétés, (16), 51-55.

Navarro, M., Martí, A., Aznar, J., Martínez, I., y De Miguel, M. (2008). Situación de la mujer en el medio rural de la Región de Murcia. Memoria, Instituto de la Mujer de la Región de Murcia. Recuperado de: https://www.carm.es/web/pagina?IDCONTENIDO=7376\&IDTIPO=246\&RAST $\mathrm{RO}=\mathrm{c} 890 \$ \mathrm{~m} 23040(18 / 10 / 2018)$.

Nieto, A., y Cárdenas, G. (2017). 25 años de políticas europeas en Extremadura: turismo rural y método LEADER. Cuadernos de Turismo, (39), 389-416. Recuperado de: https://revistas.um.es/turismo/article/view/290621 (22/01/2020).

Office of The Royal Development Projects Board (2017). Kung Krabaen Bay Royal Development Study Centre. Recuperado de: http://www.rdpb.go.th/en $(22 / 01 / 2020)$.

OMT (2019). Panorama del turismo internacional, 23. Recuperado de: https://www.eunwto.org/doi/pdf/10.18111/9789284421237 (22/01/2020).

Pecqueur, B. (2005). Le développement territorial : une nouvelle approche des processus de développement pour les économies du Sud. En A. Benoît, et F. Giraut (eds.), Le territoire est mort. Vive les territoires!: une refabrication au nom du développement (pp. 295-316). IRD Éditions. Recuperado de: https://horizon.documentation.ird.fr/exl-doc/pleins_textes/divers1007/010035257.pdf (08/04/2019).

Pérez, O. A. (2014). Subversión feminista de la economía. Aportes para un debate sobre el conflico capital-vida. Traficante de Sueños.

Pulido, J. I., y Cárdenas, P. J. (2011). El turismo rural en España. Orientaciones estratégicas para una tipología aún en desarrollo. Boletín de la Asociación de Geógrafos Españoles, (56), 155-176. Recuperado de: https://bage.agegeografia.es/ojs/index.php/bage/article/view/1348/1271 (08/04/2019).

Pulido, J. I., y Parrilla, J. A. (2016). ¿Influye el dinamismo económico del turismo en el desarrollo socioeconómico de un territorio? Un análisis mediante ecuaciones estructurales. Revista de Estudios Regionales, (107), 87-120. Recuperado de: http://www.revistaestudiosregionales.com/documentos/articulos/pdf-articulo2505.pdf (17/04/2020). 
Ramos, E., y Garrido, D. (2011). Desarrollo Rural Territorial: Metodología de Aplicación para el Estudio de Casos. Ministerio de Medio Ambiente y Medio Rural y Marino. Centro de Publicaciones.

Rijkers, B., y Costa, R. (2012). Gender and Rural Non-Farm Entrepreneurship. World Development, 40(12), 2411-2426. https://doi.org/10.1016/j.worlddev.2012.05.017.

Rubio M. A., y Mazón, T. (2009). El capital social como factor coadyuvante de los procesos de desarrollo turístico y socioeconómico de los destinos de interior. Papers de Turisme, (45), 41-55. Recuperado de: http://www.papersdeturisme.gva.es/ojs/index.php/Papers/article/view/72 $(17 / 04 / 2020)$.

Saarinen, J. (2004). Destinations in change. Tourist Studies, 4(2), 161-179. https://doi.org/10.1177/1468797604054381.

Sánchez-Oro, M., y Pérez Rubio, J. A. (2007). Aproximación a las percepciones y orientaciones de los jóvenes ante el futuro del medio rural en Extremadura. Política y Sociedad, 44(3), 195-217. Recuperado de: https://revistas.ucm.es/index.php/POSO/article/view/POSO0707330195A $(18 / 10 / 2018)$.

Santos, X. M., y Lois, R. C. (2015). Planificación y espontaneidad en el desarrollo rural. En E. Pérez, y R. Rodríguez (eds.), Espacios y desarrollos rurales. Una visión múltiple desde Europa y Latinoamérica (pp. 131-156). Ediciones Trea.

Saraniemi, S., y Kylänen, M. (2011). Problematizing the Concept of Tourism Destination: An Analysis of Different Theoretical Approaches. Journal of Travel Research, 50(2), 133-143. https://doi.org/10.1177/0047287510362775.

Sarasa, A. (2000). Aportaciones Básicas del Turismo al Desarrollo Rural. Cuadernos de Turismo, 6, 45-59. Recuperado de: https://revistas.um.es/turismo/article/view/22661 (06/08/2018).

Seuneke, P., y Bock, B. B. (2015). Exploring the roles of women in the development of multifunctional entrepreneurship on family farms: An entrepreneurial learning approach. NJAS - Wageningen Journal of Life Sciences, (74-75), 41-50. https://doi.org/10.1016/j.njas.2015.07.001.

Shiva, V., y Mies, M. (2016). Ecofeminismo. Icaria.

Shiva, V. (1998). Abrazar la vida. Mujer, ecología y supervivencia. Horas y Horas.

Shortall, S. (2015). Gender mainstreaming and the Common Agricultural Policy. Gender, $\begin{array}{llll}\text { Place } \quad \text { and } & \text { Culture, }\end{array}$ https://doi.org/10.1080/0966369X.2014.939147.

Shortall, S., McKee, A., y Sutherland, L. A. (2020). The Performance of Occupational Closure: The Case of Agriculture and Gender. Sociologia Ruralis, 60(1), 40-57. https://doi.org/10.1111/soru.12279.

Sparrer, M. (2003). Género y turismo rural. El ejemplo de la costa coruñesa. Cuadernos de Turismo, (11), 181-197. Recuperado de: https://revistas.um.es/turismo/article/view/19441 (03/02/2020).

Thuesen, A. A. (2010). Is LEADER elitist or inclusive? Composition of danish lag boards in the 2007-2013 rural development and fisheries programmes. Sociologia Ruralis, 50(1), 31-45. https://doi.org/10.1111/j.1467-9523.2009.00500.x. 
Vázquez-Barquero, A (2007). Desarrollo endógeno. Teorías y políticas de desarrollo territorial. Investigaciones Regionales: Journal of Regional Research, (11), 183210. Recuperado de: https://www.redalyc.org/pdf/289/28901109.pdf (08/04/2019).

Vázquez-Barquero, A. (2017). Dinámica productiva y desarrollo en las áreas rurales. Panorama Económico, 25(3), 289-296. https://doi.org/10.32997/2463-0470vol.25-num.3-2017-2077.

Vázquez-Barquero, A. (2018). Reflexiones teóricas sobre la relación entre desarrollo endógeno y economía social. Revista Iberoamericana de Economía Solidaria e Innovación Socioecológica, (1), 11-22. Recuperado de: http://rabida.uhu.es/dspace/bitstream/handle/10272/15749/reflexiones_teoricas.pd f? sequence $=2(17 / 04 / 2020)$.

Woods, M. (2005) Rural geography: processes, responses and experiences in rural restructuring. Sage. http://dx.doi.org/10.4135/9781446216415.

Wright, W., y Annes, A. (2014). Farm Women and Agritourism: Representing a New Rurality. Sociologia Ruralis, 54(4), 477-499. https://doi.org/10.1111/soru.12051. 


\section{CONTRIBUCIÓN SEGÚN AUTORES}

\begin{tabular}{|c|l|c|c|c|}
\hline \multicolumn{1}{|c|}{ ITEM } & César García & $\begin{array}{c}\text { M del Carmen } \\
\text { Solano }\end{array}$ & $\begin{array}{c}\text { Prudencio J. } \\
\text { Riquelme }\end{array}$ \\
\hline 1 & Conceptualización & $50 \%$ & $30 \%$ & $20 \%$ \\
\hline 2 & Tratamiento de los datos & $0 \%$ & $0 \%$ & $0 \%$ \\
\hline 3 & Análisis formal & $10 \%$ & $50 \%$ & $40 \%$ \\
\hline 4 & Acceso financiación & $0 \%$ & $0 \%$ & $0 \%$ \\
\hline 5 & Investigación & $60 \%$ & $25 \%$ & $15 \%$ \\
\hline 6 & Metodología & $50 \%$ & $35 \%$ & $15 \%$ \\
\hline 7 & Gestión del proyecto & $30 \%$ & $30 \%$ & $40 \%$ \\
\hline 8 & Recursos & $0 \%$ & $0 \%$ & $0 \%$ \\
\hline 9 & Software & $0 \%$ & $0 \%$ & $0 \%$ \\
\hline 10 & Supervisión & $0 \%$ & $0 \%$ & $0 \%$ \\
\hline 11 & Validación & $0 \%$ & $0 \%$ & $0 \%$ \\
\hline 12 & Visualización & $70 \%$ & $30 \%$ & $0 \%$ \\
\hline 13 & Redacción (borrador) & $80 \%$ & $10 \%$ & $10 \%$ \\
\hline 14 & Redacción final (revisión y edición) & $50 \%$ & $25 \%$ & $25 \%$ \\
\hline
\end{tabular}

Para más información, visitar CRediT: https://casrai.org/credit/ 